\title{
Controlled Folding of Graphene: GraFold Printing
}

Toby Hallam, ${ }^{* \dagger}{ }^{\dagger}$ Amir Shakouri, ${ }^{\ddagger}$ Emanuele Poliani, ${ }^{\S}$ Aidan P. Rooney, Ivan Ivanov, ${ }^{\perp}$ Alexis Potie, ${ }^{\dagger}$ Hayden K. Taylor, ${ }^{\#}$ Mischa Bonn, ${ }^{\perp}$ Dmitry Turchinovich, ${ }^{\perp}$ Sarah J. Haigh, ${ }^{\|}$Janina Maultzsch, ${ }^{\S}$ and Georg S. Duesberg ${ }^{\dagger, \nabla}$

\author{
${ }^{\dagger}$ Centre for Research on Adaptive Nanostructures and Nanodevices (CRANN) and Advanced Materials BioEngineering Research \\ Centre (AMBER), and ${ }^{\nabla}$ School of Chemistry, Trinity College, Dublin 2, Ireland \\ ${ }^{\ddagger}$ Nanyang Technological University, 50 Nanyang Ave, Singapore 639798, Singapore \\ ${ }^{\S}$ Institut für Festkörperphysik, Technische Universität, 10623 Berlin, Germany \\ "School of Materials, University of Manchester, Oxford Road, Manchester M13 9PL, United Kingdom \\ ${ }^{\perp}$ Max Planck Institute for Polymer Research, Ackermannweg 10, 55128 Mainz, Germany \\ \#Department of Mechanical Engineering, University of California, Berkeley 94720, United States
}

\section{Supporting Information}

ABSTRACT: We have used elastomeric stamps with periodically varying adhesive properties to introduce structure and print folded graphene films. The structure of the induced folds is investigated with scanning probe techniques, high-resolution electron-microscopy, and tip-enhanced Raman spectroscopy. Furthermore, a finite element model is developed to show the fold formation process. Terahertz spectroscopy reveals induced anisotropy of carrier mobility along, and perpendicular to, the graphene folds. Graphene fold printing is a new technique which allows for significant modification of the properties of $2 \mathrm{D}$ materials without damaging or chemically modifying them.

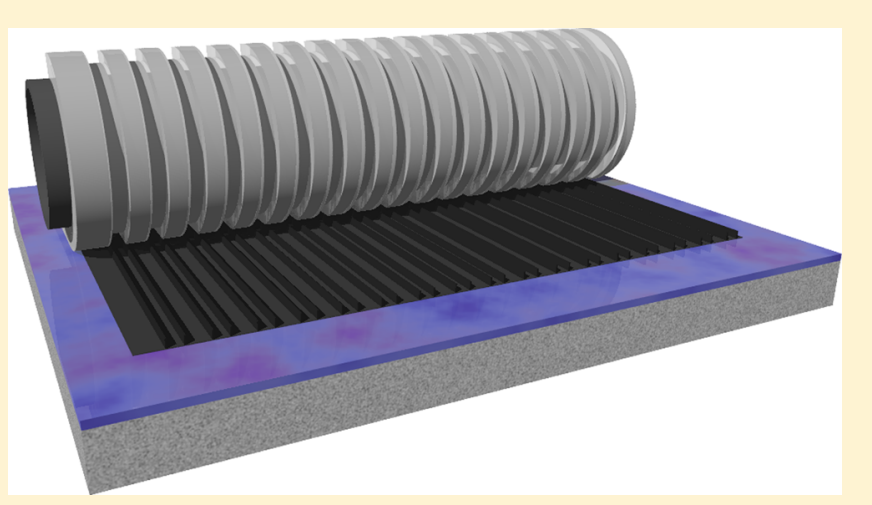

KEYWORDS: Graphene, patterning, folding, contact printing, nanotechnology, spectroscopy

T $\mathrm{n}$ the past few years, significant inroads have been made into shaping, patterning, doping, and processing of graphene to create novel technological applications. ${ }^{1}$ However, such approaches have generally ignored the ability of flat $2 \mathrm{D}$ materials to fold and crease. In fact, such deformations are typically considered to be undesirable. ${ }^{2}$ This is surprising, since the ability to fold graphene is actually one of the few properties that are entirely unique to $2 \mathrm{D}$ materials, a truly new avenue for material processing.

Due to an intrinsic compressive strain in chemical vapor deposited (CVD) graphene and its atomically thin nature, folds occur spontaneously. ${ }^{3-5}$ The properties of these folds have been isolated, and in some cases folds have been intentionally created. ${ }^{6,7}$ But, the nature of these investigations is limited due to the complicated experimental configuration required to investigate naturally occurring folds or to induce them.

The ability to controllably form folds in graphene has significant research and technological applications. Induced folds have a sublithographic width and macroscopic length. They could be used as channel materials or interconnects in chips, and it has been shown that stable field emitters are formed by folded graphene. ${ }^{8}$ Besides the morphological characteristics, the electronic structure is also affected. Due to the symmetry breaking in the graphene lattice, folding can locally enhance the spin orbit coupling of the material leading to pseudomagnetic fields. ${ }^{9,10}$ Folding of graphene also causes rehybridization of the $\mathrm{sp}^{2}$ bond network leading to a more $\mathrm{sp}^{3}$ type character which allows for covalent chemistry to be carried out on the usually unreactive graphene surface. ${ }^{11,12}$

A method for controlled fold introduction could allow for the harnessing of these types of effects for technological advantage. Compared to polymer-assisted transfer, printed folds have a unique relaxed strain profile and provide a platform for locally suspended CVD graphene. Furthermore, controlled arrays of folds have recently been predicted to have beneficial tertiary effects such as spin polarization and bandgap opening. ${ }^{13}$

These applications are only those which are most obvious in graphene. The past few years have introduced a cornucopia of two-dimensional materials such as h-BN, $\mathrm{MoS}_{2}$, and $\mathrm{WSe}_{2}$ to name but a few. Polymer-assisted transfer has been applied to transfer CVD layered materials ${ }^{14}$ and should be directly adaptable to the GraFold process. Other layered materials

Received: September 9, 2014

Revised: December 17, 2014 
a)

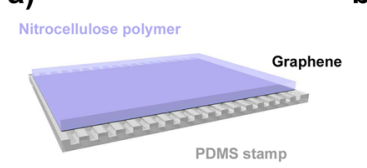

"inking" b)

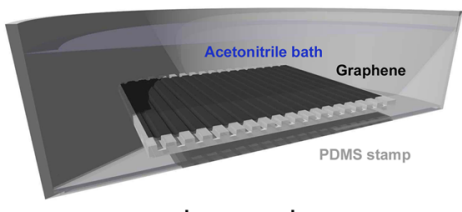

graphene release

c)

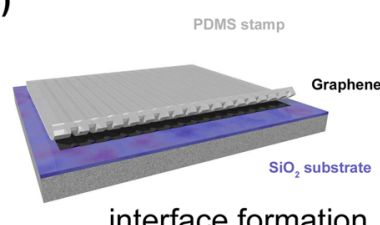

d)

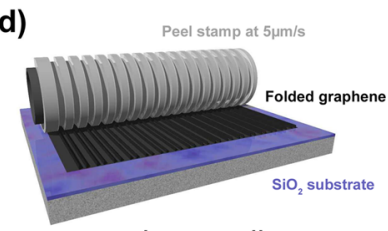

slow peeling

Figure 1. Schematic representation of the GraFold concept. (A) A polymer supported graphene film is draped across a relief patterned stamp. (B) Polymer layer is dissolved. (C) The stamp is placed onto the substrate, and PDMS is separated from graphene by slow peeling. (D) Collapse of graphene loops creates free-standing folds.

give us exciting material properties that may be tuned with folding such as band gap engineering, spin and valley degrees of freedom, and heterostructure interactions. ${ }^{15}$

In this work we demonstrate a controlled graphene folding process (GraFold) using a microcontact printing method. Graphene is transferred to a patterned elastomeric stamp and mechanically folded by placing the stamp onto a destination substrate and slowly peeling it away. A low rate of peeling is used such that graphene is released by the elastomer and transferred to the substrate. We explore the structure of these artificial folds and elaborate on the fold formation mechanism. We also show that, by this simple method, charge transport anisotropy may be induced in graphene.

We would like to note that in this work and previously ${ }^{8}$ we have used the term "fold" to indicate an out-of-plane deformation created by the GraFold printing process. The folds created here are different from wrinkles detailed by Zhu et al. ${ }^{6}$ but do not contain interfacial stacking as described by Jiang et al. ${ }^{16}$ As such they are a distinct, induced entity.

Results and Discussion. As previously intimated, GraFold is a transfer printing process. The excess graphene required for forming the folds is induced by using polydimethylsiloxane (PDMS) stamps with a relief pattern such that the graphene tension and adhesion is modulated across the stamp. Initially the graphene is kept in a rigid planar structure by a polymer support layer. This stage is the "inking" of the stamp, shown in Figure 1a.

When the polymer layer is dissolved with an appropriate solvent, the graphene is able to relax into the recessed features and partially adhere to the sidewalls as shown in Figure $1 \mathrm{~b}$. This sidewall adhesion is enhanced during the drying process.

The graphene inked stamp is then placed gently onto the destination substrate, and a conformal contact is made between the stamp, graphene, and substrate. The stamp is then slowly peeled away leaving the mechanically patterned graphene film attached to the substrate. The peeling direction is parallel to the fold features in order to limit tearing. For similar reasons the peel rate should not exceed $15 \mu \mathrm{m} / \mathrm{s}$, with a typical a peel rate being $2-5 \mu \mathrm{m} / \mathrm{s}$. The origin of this tearing is flaws such as grain boundaries failing in free-standing graphene as the stamp is removed. Different stamp dimensions or material quality should change achievable print speeds. An optical micrograph of a printed film with an undesirably fast peel speed of $25 \mu \mathrm{m} / \mathrm{s}$ is shown in Supplementary Figure Sla exhibiting no induced folds and extensive tearing.

The graphene films printed by the GraFold process exhibit periodic line features as shown in Figure 2a. The line structures persist across the surface and are aligned with the patterned features on the stamp. The graphene and the features are visible optically with a $100 \times$ confocal light microscope on $300 \mathrm{~nm}$ $\mathrm{SiO}_{2}$ as this is a high-contrast configuration for graphene observation. On other surfaces such as metal surfaces where no optical interference effect exists, the folded graphene film is invisible indicating the cleanliness of the folded graphene. ${ }^{17}$ The optical micrographs also reveal that there is some tearing of the graphene film. This tearing is due to large forces on the graphene during solvent drying and printing. ${ }^{8}$ When printing continuous films, extensive tearing is unavoidable, and to mitigate this, controlled boundaries are introduced into the graphene by etching of the graphene before the stamp is inked. The influence of etching graphene for strain mitigation is discussed in Supporting Information S1 and subsequently in Supporting Information S4.6.

Scanning electron microscopy (SEM) in Figure $2 \mathrm{~b}$ reveals the structure of the folds more exactly, indicating that the folds have some imperfections in their structure and occasionally interact with other intrinsic folds, the presence of which may cause them to diverge slightly from their course or be locally suppressed. SEM images also show some tearing of the graphene films as with optical microscopy.

Atomic force microscopy (AFM) has also been used to characterize GraFold films. We have used high aspect ratio supersharp AFM tips to minimize convolution of the topography with the AFM probe dimensions. The image in Figure $2 c$ shows an area of film containing two folds. The inset shows that the height of the folds is $\sim 15 \mathrm{~nm}$. For the specific process conditions shown in this paper the height of generated folds is $17.9 \mathrm{~nm}$ with a standard deviation of $4.2 \mathrm{~nm}$ (from 60 measurements). However, under different conditions folds have also been created with heights over $100 \mathrm{~nm}$ (discussion in Supporting Information S1). The AFM further shows some bubbles in-between the folds where the graphene does not sit perfectly flat on the substrate. This type of behavior is often observed for graphene that has been transferred. ${ }^{5}$

While AFM gives some notion of the lateral extent of the fold features, it is necessary to use cross-sectional high angle annular dark field (HAADF) Scanning transmission electron microscope (STEM) imaging to visualize the exact structure of the created folds. It has been previously applied to reveal structural information for individual atomic layers buried in graphene-BN heterostructure devices. ${ }^{18}$ For that a focused ion beam was used to cut a cross sectional lamella perpendicular to the printed graphene fold, which is protected by depositing carbon and metal layers. Comparison of fold dimensions from SEM before and after the addition of the protective carbon and platinum layers shows that the folds do not change significantly confirming that the cross sectional STEM image shown in Figure $2 \mathrm{~d}$ is representative of the folds' native configuration.

The graphene fold was not directly observed for the present structure using either TEM or STEM imaging. This is likely to be a consequence of small irregularities in the graphene fold which mean that the graphene sheet is not locally oriented 

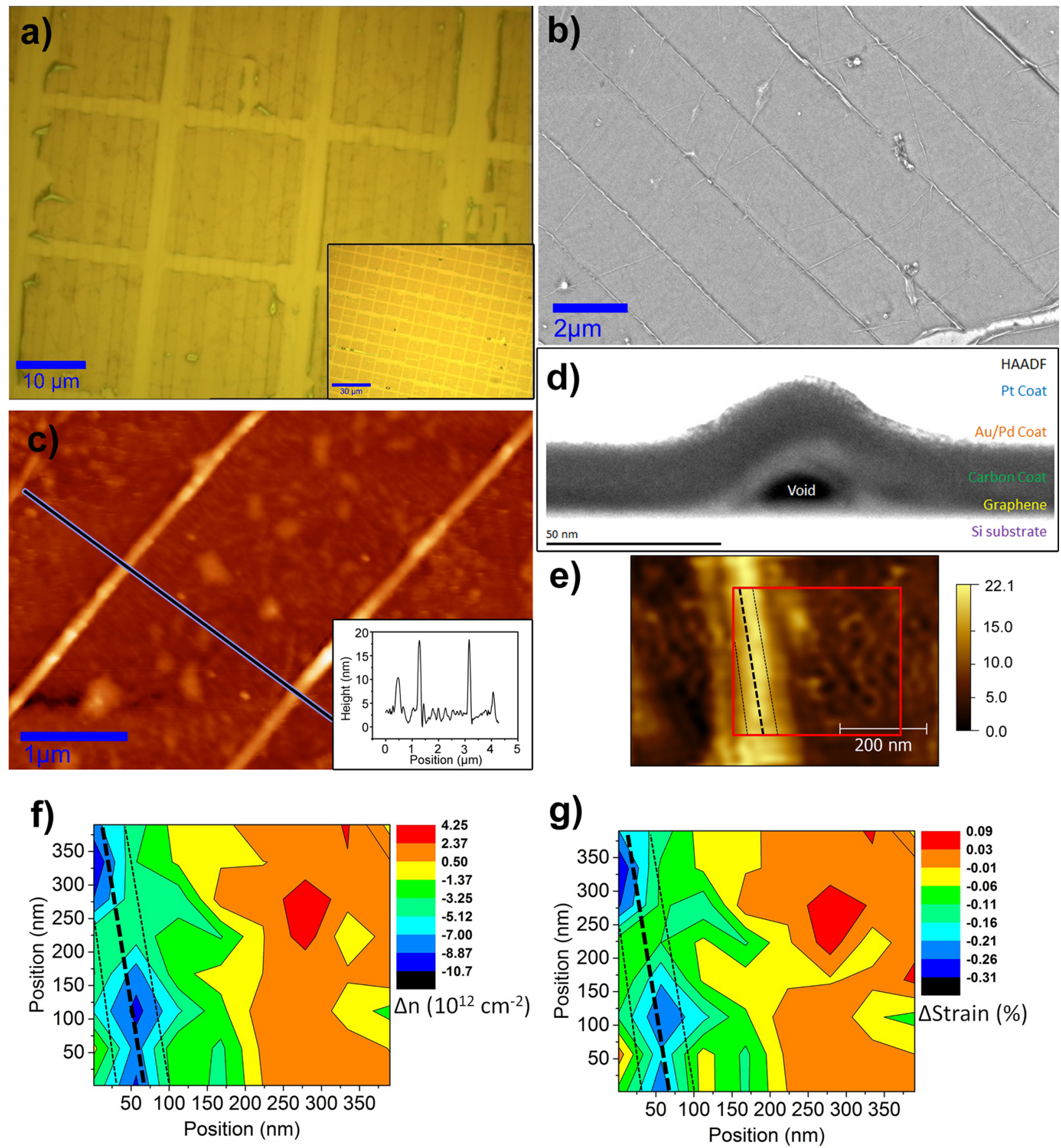

Figure 2. Microscopic characterization of the folded graphene film. (A) Optical micrograph of folded graphene film. The inset shows a large scale image of the film indicating the low level of tearing. (B) SEM of a folded graphene film. (C) AFM of a folded graphene film. Topography of the region under the blue line is shown as a linescan inset. (D) Cross-sectional HAADF STEM of graphene fold. (E) AFM image of the single fold. The region studied with TERS is marked with the solid red line. (F) Contour map for graphene doping variation extracted from TERS data, assuming hole doping. (G) Contour map for graphene strain variation extracted from TERS data. The dashed black lines in E, F, and G indicate the region of the fold. (The Raman maps corresponding to F and G are presented in Supporting Information S3.)

perfectly perpendicular to the electron beam and therefore shows reduced contrast. Nevertheless, the location and shape of the graphene fold can be located using energy dispersive X-ray (EDX) spectrum imaging in the STEM to elemental map the position of the graphene layer from the presence of residual chromium on the graphene surface as shown in the Supporting Information, Section S2. The presence of trace chromium stems from a chromium stabilizing layer found on the copper foil used for graphene growth. The void underneath the graphene ridge is thought to have been absent of material before the preparation of the lamella but now contains a small amount of carbon and silicon redeposited during FIB milling. Together the elemental maps and HAADF STEM images reveal the morphology of printed folds, identifying a radius of curvature approximately equal to the height, i.e., $20 \mathrm{~nm}$. The radius of curvature of the printed folds of is therefore too big to induce carbon nanotube-like behavior such as a bandgap opening or splitting of the G mode. ${ }^{19}$ 

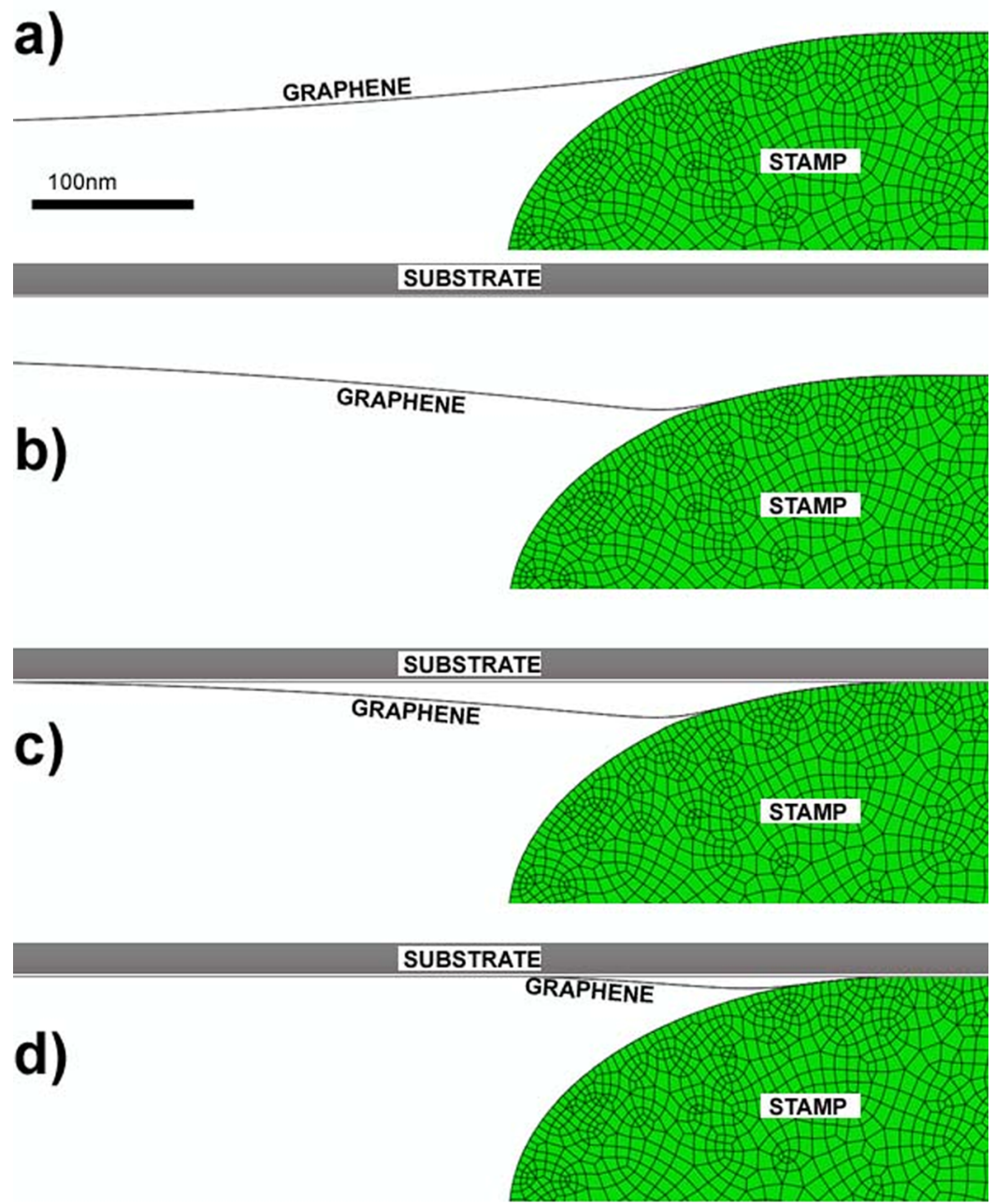

Figure 3. Finite element model for graphene fold formation. (A) Artificial "inking" of graphene onto the stamp. (B) Relaxed inked configuration. (C) Response of the system to contact with a substrate. (D) Final configuration of the system after relaxation. The scale bar shown in A is common for all parts.

In addition to the structure we are able to retrieve information about strain and doping with Raman spectroscopy. It is well-known that strain, as it changes the lattice constants, can be monitored by Raman spectroscopy in graphene. ${ }^{20-22}$ However, variation in charge carrier density in graphene also shifts phonon frequencies due to electron-phonon coupling effects. $^{23,24}$ In order to disentangle strain and doping information in our folded graphene films we have adapted the vector decomposition method introduced by Lee et al. ${ }^{21}$ (see also S3). In order to see the behavior of individual fold elements, we have used near-field tip-enhanced Raman spectroscopy (TERS), allowing for hyperspectral Raman mapping with sub wavelength lateral resolution. ${ }^{25,26}$

With the TERS measurements we were able to track the Raman $G$ and 2D-mode frequencies across a printed fold. Assuming that CVD-grown and exfoliated graphene own a similar dependence of the G and 2D modes on strain and holedoping, we estimate the variations in doping and strain from the $\mathrm{G}$ and 2D-mode positions in the TERS map (see S4). The extracted maps of local doping and strain variation are presented in Figure $2 \mathrm{e}$ and $\mathrm{f}$, where the black lines indicate the region of the fold. Figure $2 \mathrm{e}$ shows that the region of the fold exhibits reduced doping density when compared to the flat regions around the fold as reference values. The doping density at the tip of the fold is different to that on $\mathrm{SiO}_{2}$ by $10^{12} \mathrm{~cm}^{-2}$, which is consistent with the typical value of $p$-type doping of graphene by $\mathrm{SiO}_{2}$ substrates. ${ }^{27}$ This can be understood as reduced doping in the fold as it is not in contact with the electrically active substrate. ${ }^{24}$ This also supports the STEM findings that the folds do not contain intercalated material, which would locally dope the graphene. Given this low level of doping in the graphene, printed folds provide an effective method of generating locally suspended graphene.

Figure $2 \mathrm{f}$ shows the same region with the extracted strain information. The fold region shows a local variation of up to $+0.3 \%$ compressive strain from holding the out-of-plane fold deformation. A rough estimate of the absolute residual strain in the surrounding regions can be done by comparison with the Raman frequencies determined for undoped and unstrained exfoliated graphene (to the best of our knowledge, no data on undoped and unstrained CVD graphene have been reported so far). This shows around $0.1 \%$ compressive strain for the regions around the folds. A number of additional measurements with confocal Raman spectroscopy over different flat regions on the 
same sample indicate even smaller strain values. This contrasts with other reports of compressive strain in transferred CVD graphene where the entire film is under strain. ${ }^{5,28}$ For printed graphene, the intrinsic compressive strain is released by draping graphene on the flexible PDMS stamp before transferring to the substrate.

The Raman measurements indicated that the folds created by the graphene printing process are different to the transferinduced wrinkles generated by the release of compressive strain during polymer assisted transfer. In order to shed additional light on the fold generation, we have carried out a simulation of the GraFold process with a static geometrically nonlinear finite element model (FEM). We have based the stamp dimensions on those taken from AFM topographical information, and the adhesive parameters of stamp and surface are taken from AFM indentation experiments (see Supplementary Discussion S4).

The simulation, shown in Figure 3, is initialized after the "inking" step; e.g., we have artificially inked the stamp by mechanically pulling the graphene film down into the trench. Then, the downward traction is removed, the system is left to relax, and the graphene film adheres onto the stamp surface such that the system is artificially "inked". The inked surface is then brought into contact with the substrate, and once again the system is allowed to relax. At this stage we can see from the simulation that a fold has been initiated. Following separation of the stamp and substrate, the fold remains on the substrate. The dimensions of this fold are consistent with those observed in cross-sectional STEM.

We can see from the FEM in Figure 3 that the process of fold initiation can be explained as a competition between relatively strong short-range graphene-substrate adhesion and weak long-range graphene-stamp adhesion. In our model we have modified the stamp geometry for a number of cases to investigate the effect of stamp curvature on the initiation of folds. In Supplementary Section S4.5 we show that as the radius of curvature of the stamps ridge edges increases from 0.2 to 0.3 $\mu \mathrm{m}$, so does the height of the initiated fold. Folds initiation is, however, not observed to be very sensitive to the exact topography of the ridge edge: fold initiation is also observed through simulation for the case of varying-curvature topography (see Supplementary Figure S7) with the average curvature radius close to that of the stamp used in the experiment.

The FEM also shows (Supplementary Section S4.6) that the forces on the graphene during the printing process are large across the trenches on the stamp. This corresponds with our observations of printing continuous folded films which often showed extensive tearing. To minimize this uncontrolled tearing, we etch the graphene into smaller regions before printing as shown in Figure 1a. This reduces the forces during the solvent evaporation and allows for a small amount of lateral slipping of the graphene across stamp. FEM shows that lateral slippage of the graphene films reduces the forces during printing significantly, and as a result folded graphene films can be printed over large regions with minimal tearing. Full details regarding the FEM model are presented in Supplementary Section S4.

In order to study the influence of the GraFold process on carrier transport properties in graphene, we have used ultrafast terahertz $(\mathrm{THz})$ spectroscopy. This technique allows one to access the conductivity dynamics in materials under test in a noncontact fashion. The linearly polarized single-cycle, subpicosecond pulses of $\mathrm{THz}$ radiation serve as sensitive conductivity probes. ${ }^{29}$ The measurement of the transmission through the sample of $\mathrm{THz}$ pulses polarized parallel or perpendicular to the folds allowed us to measure the conductivity of folded graphene along these two orthogonal directions, as explained below. We note here that, similarly to other photoconductive materials, ${ }^{29}$ in graphene the distinction should be made between the $\mathrm{THz}$ frequency mobility and the dc mobility. The $\mathrm{THz}$ radiation has submm wavelength, and consequently the $\mathrm{THz}$ beam spot on the sample was on the order of $1 \mathrm{~mm}$, much larger than any nano- or microscopic features of our folded graphene sample (Figure $2 \mathrm{a}$ and $\mathrm{b}$ ). Hence, the area-averaged conductivity of the folded graphene was probed using $\mathrm{THz}$ spectroscopy. In our experiments we have measured the transient conductivity of folded graphene upon the photoexcitation with 100 fs laser pulses of $800 \mathrm{~nm}$ wavelength (1.55 eV photon energy), as illustrated in Figure 4a. Such a optical pump-THz probe

a)
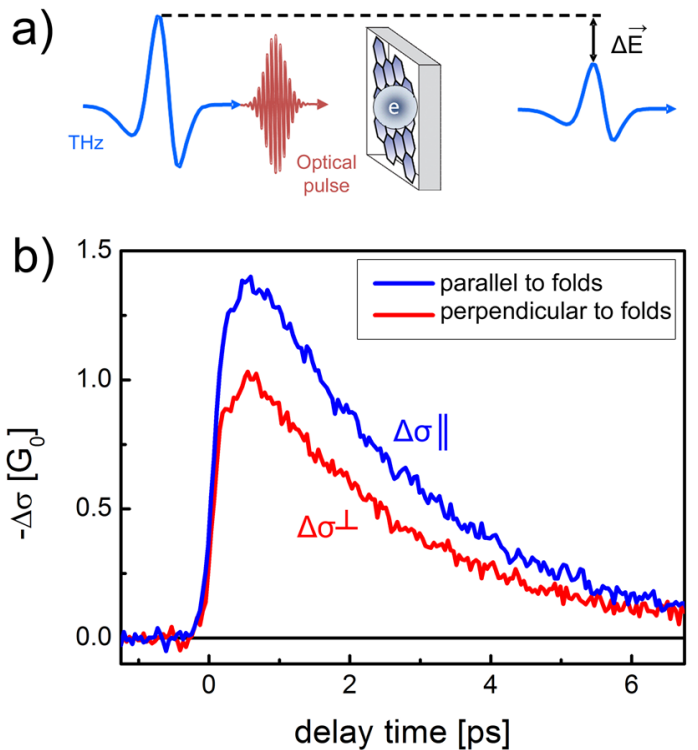

Figure 4. Fold-induced anisotropy in charge carrier mobility. (A) Optical $(800 \mathrm{~nm}, 100 \mathrm{fs})$ pump-THz probe measurement shown schematically. (B) Modulation in $\mathrm{THz}$ conductivity as a function of pump-probe delay, measured for the $\mathrm{THz}$ field polarized parallel and perpendicular to the directions of folds, and expressed in quantum units of $G_{0}=\mathrm{e}^{2} / 4 \hbar=6.04 \times 10^{-5} \mathrm{~S}$. As there is no pump absorption anisotropy, the anisotropy of $\mathrm{THz}$ photoconductivity measured parallel and perpendicular to the folds is due to the anisotropy of electron mobility along these two directions.

(OPTP) method allows for background-free, quantitative conductivity measurements on an ultrafast time scale. ${ }^{30}$ The relaxation of initially photogenerated carriers in graphene is known to occur on a similar time scale as their photoexcitation of $\sim 100 \mathrm{fs}^{31}$ While relaxing, the photogenerated carriers in turn transfer a significant part of their energy to the background electrons present in graphene due to substrate doping (see Figure 2e), resulting in electron gas heating. ${ }^{30}$ This heating of background electron population in turn results in the reduction of intrinsic (i.e., in the absence of photoexcitation) $\mathrm{THz}$ frequency mobility for these hot carriers, consequently leading to the reduction of the $\mathrm{THz}$ conductivity of photoexcited graphene $-\Delta \sigma$, which is shown in Figure $4 \mathrm{~b}$. The relaxation of $-\Delta \sigma$ on the time scale of ca. 10 ps reflects the dynamics of electron gas cooling, which occurs via phonon emission. ${ }^{30}$ As can be seen in Figure $4 b$, the $1.4: 1$ ratio of the $\mathrm{THz}$ photoconductivity measured parallel and perpendicular to the 
folds is observed in folded graphene. As no optical pump absorption anisotropy was found in our sample which could lead to the asymmetry in the density of photoexcited charge $N$, the observed anisotropy of photoconductivity $-\Delta \sigma=\mu \mathrm{Ne}$ can only be related to the anisotropy of electron mobility in the direction parallel and perpendicular to the folds of $\mu_{||} / \mu_{\perp}=1.4$. TERS measurements (see Figure $2 \mathrm{f}$ ) reveal the difference in local doping densities on the order of $10^{13} \mathrm{~cm}^{-2}$ between the folds and surrounding areas, which must result in substantial local potential fluctuations in the direction perpendicular to the folds. Therefore, the most likely cause for the observed carrier mobility anisotropy is a highly anisotropic potential landscape for the carriers moving perpendicular and parallel to the folds. We note that in our samples the $100 \mathrm{~nm}$ folds of lightly doped graphene are separated by $2 \mu \mathrm{m}$ stripes of highly doped one (Figures $2 \mathrm{~b}, \mathrm{e}$ ), and hence the folds comprise only about $5 \%$ of the area of the THz-probed sample. In this respect, it is quite remarkable that such a low filling fraction of $1 \mathrm{D}$ folds results in a conductivity anisotropy as high as $1.4: 1$, clearly indicating the potential of GraFold for optoelectronics applications where ultrafast directional charge transport may be required, such as in mechanically printed electronic circuits.

Conclusion. In summary, we have presented GraFold, a novel method for transferring graphene to an arbitrary substrate with periodic, introduced folds. The shape of the introduced graphene folds is a semicircular protrusion from the surface with no intercalated material. Within the semicircular folded region, the graphene shows little to no doping and a small amount of compressive strain. These properties of cleanliness and low strain make this folded graphene a type of "locally suspended" graphene that could be used to mitigate the extreme effort currently required to suspend graphene. Ultrafast optical conductivity measurements show that the periodic folding of graphene introduces significant charge transport anisotropy such that the film has a 1.4 times higher resistance in the direction perpendicular to folds. The simple pattern of printed graphene folds demonstrated in this paper is only a first expression of the GraFold technique. More complicated graphene distortions such as increased fold density, fold intersections, and graphene domes could be generated using this method. ${ }^{32-34}$ Beyond graphene, the generalization of graphene folding into other two-dimensional materials such as $\mathrm{MoS}_{2}$ is an obvious direction, suggesting that patterning of $2 \mathrm{D}$ materials by GraFold printing will be a powerful processing tool in the future.

Methods. Graphene Folding Process. CVD graphene is grown from methane by CVD on $18 \mu \mathrm{m}$ thick copper foils from Gould $\mathrm{GmbH}$, subsequently electrochemically polished in house. $^{35}$ The graphene is then transferred to a patterned PDMS stamp using a modified polymer assisted transfer with nitrocellulose (Collodion, Sigma-Aldrich) as described in refs 5 and 8. PDMS (SYLGARD 184) stamps are formed with a 5:1 polymer-cross-linker volume ratio by filling an octadecyltrichlorosilane treated $\mathrm{Si}$ master etched using $\mathrm{CF}_{4} / \mathrm{O}_{2}$ reactive ion etch (Oxford ICP) with a trench width of $2 \mu \mathrm{m}$ and a depth of $1.9 \mu \mathrm{m}$.

The polymer stabilizing layer is removed by a $3 \mathrm{~h}$ immersion in acetonitrile and a 30 min drying procedure in a solvent-rich environment. Printing was carried out with a precision translation stage (PI 403.DG) and a large diameter quartz roller moving at $1-25 \mu \mathrm{m} / \mathrm{s}$ at room temperature in a lab atmosphere.
Graphene etching into squares was achieved with a UV lithography process on graphene while still on copper foil. Etching was carried out by a $2 \mathrm{~s} \mathrm{O}_{2}$ reactive ion etch (Oxford ICP), or in the case of the transparent sample for $\mathrm{THz}$ spectroscopy, etching was carried out in an $300 \mathrm{~W}$ oxygen barrel asher at $0.3 \mathrm{mbar}$ for $2 \mathrm{~min}$.

For $\mathrm{THz}$ spectroscopy experiments folded graphene was printed onto fused silica, and in all other cases $\mathrm{SiO}_{2}(300 \mathrm{~nm}) /$ $\mathrm{Si}(100)$ wafers were used.

Microscopy. SEM images were taken with a Zeiss Supra 40 at $5 \mathrm{kV}$ acceleration voltage. SEM images have been passed through a Gaussian filter to enhance the visibility of surface detail. AFM images were taken with a Veeco Dimension 3100 AFM in tapping mode with Nanosensor super sharp cantilevers (SSS-NCH type).

Cross-Sectional STEM. A dual beam instrument (FEI Dual Beam Nova 600i) was used for site specific preparation of the cross sectional sample for STEM analysis using the lift-out approach. This instrument combines a focused ion beam (FIB), a scanning electron microscope (SEM) column, and a gasinjection system in the same chamber. This allows local material deposition and material-specific preferential milling to be performed by introducing reactive gases in the vicinity of the electron or ion probe.

High-angle annular dark field (HAADF) scanning transmission electron microscopy (STEM) was performed in a probe side aberration corrected FEI Titan G2 operated at 200 $\mathrm{kV}$ with an X-FEG electron source. A convergence angle of 26 mrad, a HAADF inner angle of $52 \mathrm{mrad}$, and a probe current of $\sim 200$ pA was used. Energy-dispersive X-ray (EDX) spectroscopy was performed using the Titan's Super-X EDX detector with a collection solid angle of $0.7 \mathrm{srad}$.

Tip-Enhanced Raman Spectroscopy (TERS). A solid gold AFM tip is illuminated by a laser which excites localized surface plasmons and its coupled evanescent field. As a consequence, the near-field excitation is spatially confined in a region determined by the typical tip-apex curvature radius, i.e., $20 \mathrm{~nm}$, and exponentially decays in the direction of the tip axis. ${ }^{26,36}$

The TERS setup consists of a Horiba-Jobin Yvon LabRam HR-800 spectrometer with $2 \mathrm{~cm}^{-1}$ spectral resolution, connected to an atomic force microscope (AFM) by a homebuilt coupling system. A $50 \times$ long-distance microscope objective, tilted $30^{\circ}$ degrees with respect to the sample plane, illuminates the tip-sample gap and collects the near-field signal. The excitation light source was a $532 \mathrm{~nm}$ laser. The AFM bulk gold tip was produced from a $50 \mu \mathrm{m}$ diameter gold wire by electrochemical etching. ${ }^{37,38}$ The hyperspectral image is formed from an $8 \times 8$ Raman map with $30 \mathrm{~s}$ acquisition time for each pixel. The distance between two adjacent pixels is 60 $\mathrm{nm}$ and gives the spatial resolution. The far-field measurement is subtracted from the tip-approached measurement in order to obtain the pure near-field signal.

$\mathrm{THz}$ Spectroscopy. The $\mathrm{THz}$ spectroscopy setup is driven by ultrafast laser pulses with central wavelength of $800 \mathrm{~nm}$, temporal length $100 \mathrm{fs}$, and pulse energy $1 \mathrm{~mJ}$. The THz pulses, produced by optical rectification in a $1 \mathrm{~mm}$ thick ZnTe crystal, were focused on the sample and then guided to the detector by the set of off-axis parabolic mirrors. Detection of the $\mathrm{THz}$ pulse transmitted through the sample was performed using an electro-optic sampling in yet another $\mathrm{ZnTe}$ crystal. The laser pulses used for the optical excitation of the folded graphene sample with $30 \mu \mathrm{J} / \mathrm{cm}^{2}$ pump fluence were derived from the same laser, and time-delayed with respect to the $\mathrm{THz}$ probe 
pulse using a scanning delay line. All the measurements were done at room temperature, in transmission configuration, and in dry nitrogen atmosphere in order to avoid the $\mathrm{THz}$ absorption by the atmospheric water vapor.

\section{ASSOCIATED CONTENT}

\section{S Supporting Information}

Additional characterization of film tearing and strain management, HAADF-STEM for GraFold features, details of TERS measurement and vector decomposition method, and details for finite-element model. This material is available free of charge via the Internet at http://pubs.acs.org.

\section{AUTHOR INFORMATION}

\section{Corresponding Author}

*E-mail: hallamt@tcd.ie.

\section{Notes}

The authors declare no competing financial interest.

\section{ACKNOWLEDGMENTS}

This work is supported by the SFI under Contract No. 12/RC/ 2278 and PI 10/IN.1/I3030. We acknowledge the EU under FP7-NMP Grafol 285275. S.J.H. and A.R. acknowledge funding from the Defense Threat Reduction Agency HDTRA1-12-10013 and Engineering and Physical Sciences Research Council NowNANO doctoral training partnership. I.I. and D.T. acknowledge EU Career Integration Grant 334324 (LIGHTER), and the Danish Council for Independent ResearchTechnology and Production Sciences (FTP project ALFIE). E.P. and J.M. acknowledge European Research Council (ERC) under grant number 259286.

\section{REFERENCES}

(1) Bonaccorso, F.; Lombardo, A.; Hasan, T.; Sun, Z.; Colombo, L.; Ferrari, A. C. Mater. Today 2012, 15 (12), 564-589.

(2) Lanza, M.; Wang, Y.; Bayerl, A.; Gao, T.; Porti, M.; Nafria, M.; Liang, H.; Jing, G.; Liu, Z.; Zhang, Y.; Tong, Y.; Duan, H. J. Appl. Phys. 2013, 113 (10), 104301-7.

(3) Liu, N.; Pan, Z.; Fu, L.; Zhang, C.; Dai, B.; Liu, Z. Nano Res. 2011, 4 (10), 996-1004.

(4) Fasolino, A.; Los, J. H.; Katsnelson, M. I. Nat. Mater. 2007, 6 (11), 858-861.

(5) Hallam, T.; Berner, N. C.; Yim, C.; Duesberg, G. S. Adv. Mater. Int. 2014, 1, 1400115.

(6) Zhu, W.; Low, T.; Perebeinos, V.; Bol, A. A.; Zhu, Y.; Yan, H.; Tersoff, J.; Avouris, P. Nano Lett. 2012, 12 (7), 3431-3436.

(7) Mei, Y.; Kiravittaya, S.; Harazim, S.; Schmidt, O. G. Mater. Sci. Eng. R-Rep. 2010, 70 (3-6), 209-224.

(8) Hallam, T.; Cole, M. T.; Milne, W. I.; Duesberg, G. S. Small 2014, 10 (1), 95-99.

(9) Levy, N.; Burke, S. A.; Meaker, K. L.; Panlasigui, M.; Zettl, A.; Guinea, F.; Castro Neto, A. H.; Crommie, M. F. Science 2010, 329 (5991), 544-7.

(10) Abedpour, N.; Asgari, R.; Guinea, F. Phys. Rev. B 2011, 84 (11), 115437.

(11) Wu, Q.; Wu, Y.; Hao, Y.; Geng, J.; Charlton, M.; Chen, S.; Ren, Y.; Ji, H.; Li, H.; Boukhvalov, D. W.; Piner, R. D.; Bielawski, C. W.; Ruoff, R. S. Chem. Commun. 2013, 49 (7), 677-679.

(12) Rasmussen, J. T.; Gunst, T.; Bøggild, P.; Jauho, A.-P.; Brandbyge, M. Beilstein J. Nano 2013, 4, 103-110.

(13) Costa, A. T.; Ferreira, M. S.; Hallam, T.; Duesberg, G. S.; Castro Neto, A. H. Europhys. Lett. 2013, 104 (4), 47001.

(14) Gurarslan, A.; Yu, Y.; Su, L.; Yu, Y.; Suarez, F.; Yao, S.; Zhu, Y.; Ozturk, M.; Zhang, Y.; Cao, L. ACS Nano 2014, 8 (11), 11522-11528.
(15) Butler, S. Z.; Hollen, S. M.; Cao, L.; Cui, Y.; Gupta, J. A.; Gutiérrez, H. R.; Heinz, T. F.; Hong, S. S.; Huang, J.; Ismach, A. F.; Johnston-Halperin, E.; Kuno, M.; Plashnitsa, V. V.; Robinson, R. D.; Ruoff, R. S.; Salahuddin, S.; Shan, J.; Shi, L.; Spencer, M. G.; Terrones, M.; Windl, W.; Goldberger, J. E. ACS Nano 2013, 7 (4), 2898-2926.

(16) Jiang, T.; Liu, H.; Huang, D.; Zhang, S.; Li, Y.; Gong, X.; Shen, Y.-R.; Liu, W.-T.; Wu, S. Nat. Nanotechnol. 2014, 9 (10), 825-829.

(17) Blake, P.; Hill, E. W.; Castro Neto, A. H.; Novoselov, K. S.; Jiang, D.; Yang, R.; Booth, T. J.; Geim, A. K. App Phys. Lett. 2007, 91 (6), 063124

(18) Haigh, S. J.; Gholinia, A.; Jalil, R.; Romani, S.; Britnell, L.; Elias, D. C.; Novoselov, K. S.; Ponomarenko, L. A.; Geim, A. K.; Gorbachev, R. Nat. Mater. 2012, 11 (9), 764-767.

(19) Reich, S.; Thomsen, C.; Maultzsch, J. Carbon Nanotubes: Basic Concepts and Physical Properties; Wiley-VCH: New York, 2004.

(20) Mohiuddin, T. M. G.; Lombardo, A.; Nair, R. R.; Bonetti, A.; Savini, G.; Jalil, R.; Bonini, N.; Basko, D. M.; Galiotis, C.; Marzari, N.; Novoselov, K. S.; Geim, A. K.; Ferrari, A. C. Phys. Rev. B 2009, 79 (20), 205433.

(21) Lee, J. E.; Ahn, G.; Shim, J.; Lee, Y. S.; Ryu, S. Nat. Commun. 2012, 3, 1024.

(22) Mohr, M.; Papagelis, K.; Maultzsch, J.; Thomsen, C. Phys. Rev. B 2009, 80 (20), 205410

(23) Bruna, M.; Ott, A.; Ijäs, M.; Yoon, D.; Sassi, U.; Ferrari, A. C. ACS Nano 2014, 8, 7432-7441.

(24) Ryu, S.; Liu, L.; Berciaud, S.; Yu, Y.-J.; Liu, H.; Kim, P.; Flynn, G. W.; Brus, L. E. Nano Lett. 2010, 10 (12), 4944-4951.

(25) Stadler, J.; Schmid, T.; Zenobi, R. Nano Lett. 2010, 10 (11), $4514-4520$

(26) Hartschuh, A. Angew. Chem., Int. Ed. 2008, 47 (43), 8178-8191.

(27) Berciaud, S.; Ryu, S.; Brus, L. E.; Heinz, T. F. Nano Lett. 2008, 9

(1), 346-352.

(28) Larsen, M. B. B. S.; Mackenzie, D. M. A.; Bøggild, P.; Booth, T. J. Microelectron. Eng. 2014, 121, 113-117.

(29) Ulbricht, R.; Hendry, E.; Shan, J.; Heinz, T. F.; Bonn, M. Rev. Mod. Phys. 2011, 83 (2), 543-586.

(30) Tielrooij, K. J.; Song, J. C. W.; Jensen, S. A.; Centeno, A.; Pesquera, A.; Zurutuza Elorza, A.; Bonn, M.; Levitov, L. S.; Koppens, F. H. L. Nat. Phys. 2013, 9 (4), 248-252.

(31) Lui, C. H.; Mak, K. F.; Shan, J.; Heinz, T. F. Phys. Rev. Lett. 2010, 105 (12), 127404.

(32) Pereira, V. M.; Castro Neto, A. H.; Liang, H. Y.; Mahadevan, L. Phys. Rev. Lett. 2010, 105 (15), 156603.

(33) Yamamoto, M.; Pierre-Louis, O.; Huang, J.; Fuhrer, M. S.; Einstein, T. L.; Cullen, W. G. Phys. Rev. X 2012, 2 (4), 041018.

(34) Levy, N.; Burke, S. A.; Meaker, K. L.; Panlasigui, M.; Zettl, A.; Guinea, F.; Neto, A. H. C.; Crommie, M. F. Science 2010, 329 (5991), 544-547.

(35) Li, X.; Cai, W.; An, J.; Kim, S.; Nah, J.; Yang, D.; Piner, R.; Velamakanni, A.; Jung, I.; Tutuc, E.; Banerjee, S. K.; Colombo, L.; Ruoff, R. S. Science 2009, 324 (5932), 1312-4.

(36) Poliani, E.; Wagner, M. R.; Reparaz, J. S.; Mandl, M.; Strassburg, M.; Kong, X.; Trampert, A.; Sotomayor Torres, C. M.; Hoffmann, A.; Maultzsch, J. Nano Lett. 2013, 13 (7), 3205-3212.

(37) Poliani, E.; Nippert, F.; Maultzsch, J. Phys. Status Solidi B 2012, 249 (12), 2511-2514.

(38) Ren, B.; Picardi, G.; Pettinger, B. Rev. Sci. Instrum. 2004, 75 (4), $837-841$. 